\title{
Drug giants target China's medical molecular biology
}

Two agreements concluded in February signified a recognition that China can offer pharmaceutical companies resources in molecular biology research and development and traditional medicine, as well as a huge market and cheap manufacturing capabilities. In March, Roche Bioscience (Palo Alto, CA) announced an agreement with two Chinese research institutes concerning the discovery of drugs for pain and lower urinary tract infections. The agreement called for the use of molecular biology to identify receptor targets, and to investige Chinese traditional medicines. Later, SmithKline Beecham (SB, King of Prussia, PA) announced its research collaboration with two other Chinese research institutes to elucidate the molecular mechanisms of disease, primarily in the areas of cardiovascular and bone disorders.

Major pharmaceutical companies have been in China for a number of years (Bio/Technology 14:151-157, 1996) but most collaborations have concerned the production and marketing of existing drugs. SB, for instance, began manufacturing operations in China in 1984, and has recently extended them to include the manufacture and marketing of Havrix, the company's hepatitis A vaccine. Other companies with biotechnological manufacturing and marketing joint ventures include Biogen (Cambridge, MA) for $\gamma$-interferon, Merck (Whitehouse Station, NJ) for hepatitis B vaccine, and Unigene Laboratories (Fairfield, NJ) for calcitonin. In February this year, Gist-brocades (Delft, the Netherlands) began a joint venture with Zhang Jia Kou Pharmaceutical (Shanghai) for the production of penicillin and Hoechst (Frankfurt), which has already invested $\$ 350$ million, plans to put another $\$ 1$ billion in by the year 2000 .

Sylvia Davidson is a freelance writer working in London.

of this program, they will spend three to six months at SB laboratories in Europe and the US, continuing basic research within SB's drug discovery program. No financial details were released.

Some smaller companies began investigating the potential of Chinese molecular biology before $\mathrm{SB}$ and Roche. Three years ago, AntiCancer (San Diego, CA) began a collaboration with Harbin Medical University (Harbin), through which the company gained access to anticancer gene therapy technology developed at the university. The most advanced of those techniques-ex vivo transfection of tumor-infiltrating lymphocytes with a vector containing the gene for interleukin-2-has already

That will include a $\$ 15.3$ million in a joint venture with the International Phatmaceutical factory (Shanghai).

Now, though, the emphasis is shifting to research. Roche will work with the Beijing Institute of Microbiology (Beijing) on the discovery and isolation of novel receptor targets, and with the Shanghai Institute of Organic Chemistry (Shanghai) on identifying new compounds based on Chinese medicinal products and the synthesis of organic compounds. The agreement stems from a long-standing "relationship" begun in the early 1990s with a group of Chinese researchers. Roger Whiting, senior vice president of the Roche Bioscience neurobiology research unit involved in the collaboration, believes that the agreements will "further strengthen the company's base in molecular biology."

SB's agreement with the National Key Laboratory of Medical Genetics (Changsha) and the Shanghai Second Medical University/Rui Jin Hospital (Shanghai) goes beyond providing financial support for research. SB will establish a training program for Chinese students and researchers from the Chinese institutes. Under the aegis entered clinical trials in China for advanced lung cancer. The technology flow is two-way. As part of the same collaboration, AntiCancer is developing in China an assay that determines the drug resistance profile of a patient's tumor cells, a technology that has already undergone trials in the US and Japan. Robert Hoffman, president of AntiCancer, said that China had not only good scientists and physicians but also "rapidly expanding commercial opportunities."

Several other US companies, including Pharmagenesis (Palo Alto, CA), Genelabs (Redwood City, CA), and Paracelsian (Itha$\mathrm{ca}, \mathrm{NY}$ ), are investigating traditional Chinese medicines. Pharmagenesis, which set up an office in China in December 1995 in collaboration with the Chinese company, President, and a provincial government body, initially aimed to isolate single pure compounds from traditional medicines. But, says the company's vice president, Robert Tidwell, it soon became clear that the mixtures themselves had different interesting properties. Pharmagenesis is due to begin clinical trials of a cancer adjuvant from a traditional Chinese medicine in China soon.

Sylvia Davidson

\section{Orphan drugs: European biotechnology waits for EC act}

The European Commission (EC, Brussels) is now putting the finishing touches on proposals for "orphan drug" legislation to be discussed in June in Brussels by national experts from all the European Union member states. Finalizing the legislative process will take at least another two years. But, although biotechnology companies have benefited considerably from orphan drug provisions in the US and Japan, biotechnology's input to the present European developments has been scarce. Accordingly, although the European proposals may benefit research into rare diseases, they may bring only limited incentives for the development of biotechnology.

Europe is the last of the major trading blocs to recognize that market forces alone cannot satisfy the needs for treatment of rare diseases. The US addressed the problem in 1983 with the Orphan Drug Act and 